J. Phys. IV France 134 (2006) 577-582

(C) EDP Sciences, Les Ulis

DOI: 10.1051/jp4:2006134089

\title{
Real-time measurement on deformation fields of hole-excavated samples under impact tension
}

\author{
Siying Chen ${ }^{1}$, Chenguang Huang ${ }^{2}$ and Guoqiang $\mathrm{Ni}^{1}$ \\ ${ }^{1}$ Department of Optoelectronic Engineering, Beijing Institute of Technology, \\ Beijing 100081, China \\ ${ }^{2}$ Department of Engineering Sciences, Institute of Mechanics, Chinese Academy \\ of Sciences, Beijing 100080, China
}

\begin{abstract}
In this paper, the real-time deformation fields are observed in two different kinds of holeexcavated dog-bone samples loaded by an SHTB, including single hole sample and dual holes sample with the aperture size of $0.8 \mathrm{~mm}$. The testing system consists of a high-speed camera, a He-Ne laser, a frame grabber and a synchronization device with the controlling accuracy of 1 microsecond. Both the single hole expanding process and the interaction of the two holes are recorded with the time interval of $10 \mu \mathrm{s}$. The observed images on the sample surface are analyzed by newly developed software based on digital correlation theory and a modified image processing method. The 2-D displacement fields in plane are obtained with a resolution of $50 \mu \mathrm{m}$ and an accuracy of $0.5 \mu \mathrm{m}$. Experimental results obtained in this paper are proofed, by compared with FEM numerical simulations.
\end{abstract}

Key words: SHPB, Digital Correlation Theory, FEM, Damage Mechanics

\section{INTRODUCTION}

The research of dynamic behaviors of materials is one of the most basic and critical part for the developments of protection engineering and defense technology. Especially, the evaluation of dynamic damage and failure at high strain rate is always a challenging and advanced subject. In this area, many achievements have been obtained in theoretical and computation aspects in recent years [1-4]. However, the improvement of testing technique is not so significant and till now in most cases the details of real local deformation are acquired by after-effect observation. A real-time and in situ measuring system is actively demanded.

In this paper, the real-time deformation fields are observed in two different kinds of hole-excavated dog-bone samples loaded by a Split Hopkinson Tension Bar (SHTB) by a transient optical measuring system, which was established in Institute of Mechanics, Chinese Academy of Sciences [5]. Both the single-hole expanding process and the interaction of the two holes are recorded with the time interval of $10 \mu \mathrm{s}$. In the following sections, the measuring system, fundamental theory and the experimental results will be shown in detail.

\section{BASIC THEORY AND MEASUREMENT SYSTEM}

From the reference [6], it is known that if we can record the difference of the surface gray-scale map during the impact process at two different times, the deformation field can be deduced by the correlation method through the following equation:

$$
C\left(u, v, \frac{\partial u}{\partial x}, \frac{\partial u}{\partial y}, \frac{\partial v}{\partial x}, \frac{\partial v}{\partial y}\right)=\frac{\sum\left[F(x, y) \cdot G\left(x^{*}, y^{*}\right)\right]}{\left[\sum\left(F(x, y)^{2}\right) \cdot \sum\left(G\left(x^{*}, y^{*}\right)^{2}\right)\right]^{\frac{1}{2}}}=\frac{\langle F \cdot G\rangle}{\left[\left\langle F^{2}\right\rangle \cdot\left\langle G^{2}\right\rangle\right]^{\frac{1}{2}}}
$$

where, $F(x, y)$ is the gray function of the first image while $G\left(x^{*}, y^{*}\right)$ denotes that after deformation. 
A picture obtained by the high-speed camera is divided into 8 subgraphs with the sequence depicted in fig.2, with the time interval of $10 \mu \mathrm{s}$. The measuring system, shown in fig.1, consists of a high-speed camera, a He-Ne laser and synchronization and recording devices.

The high-speed camera, IMACON 790, is improved by using the MTV-1881EX CCD as a recorder instead of the Polylad films. This CCD is produced by MITON company and has a resolution of 795.H.X 596.V. and an image area of $7.95 \mathrm{~mm} \times 5.08 \mathrm{~mm}$. The minimum sensitive illumination is $0.02 \mathrm{Lux}$ and the signal-to-noise performance is greater than $48 \mathrm{db}$.

Synchronizing control is very important and complicated in dynamic tests. The strain signal, which comes from a strain gauge attached to the input bar, is used as the trigger source. A three-channel synchronization device with accurate time-delay is designed to trigger the flash, high-speed camera and frame grabber in turn. The sketch map of synchronizing control is shown in Fig.3.

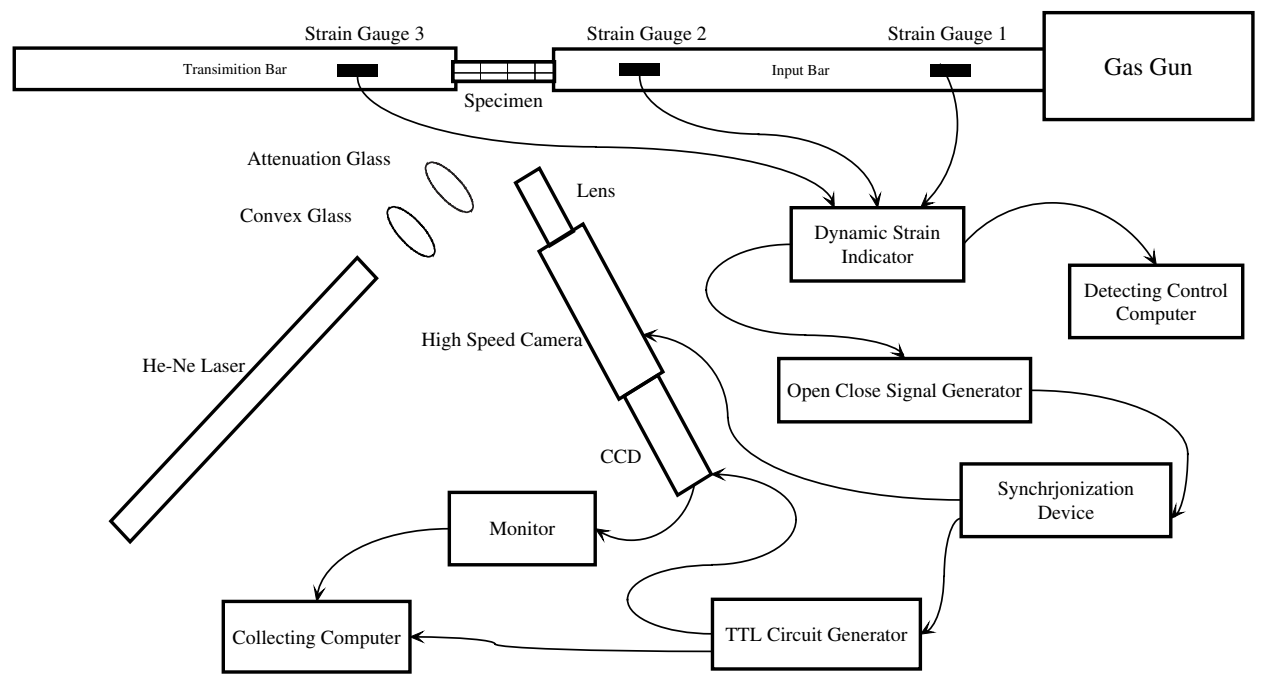

Figure 1. Sketch of measuring system.

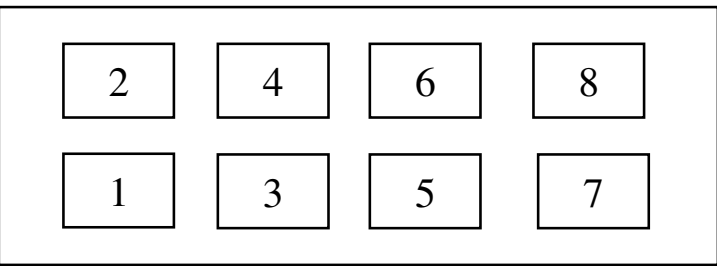

Figure 2. High-speed camera imaging sequence.

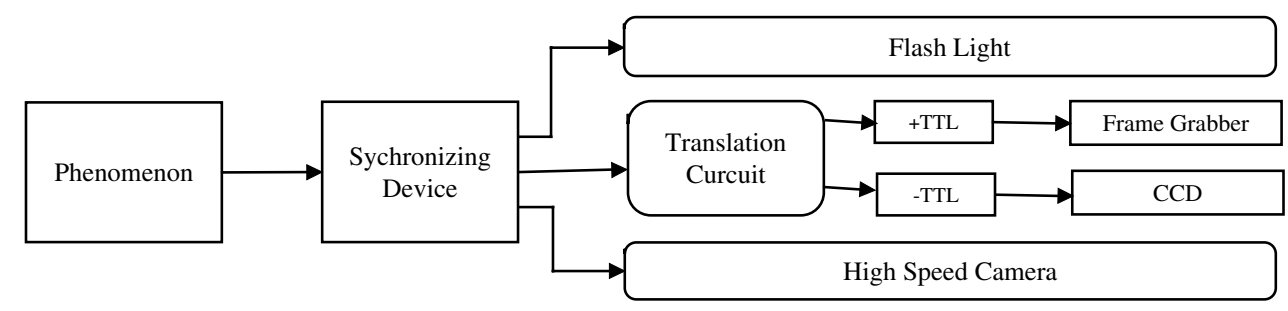

Figure 3. Synchronizing contral. 


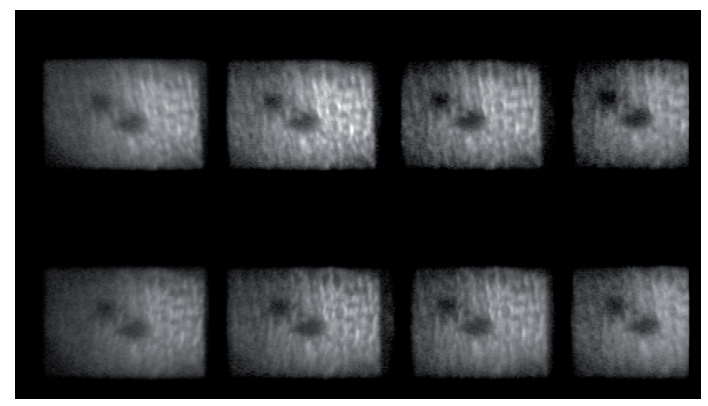

(a)

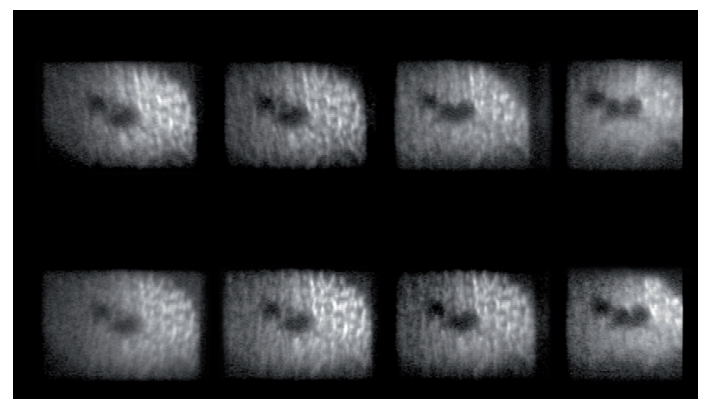

(b)

Figure 4. Images of single-hole sample before and after loading.

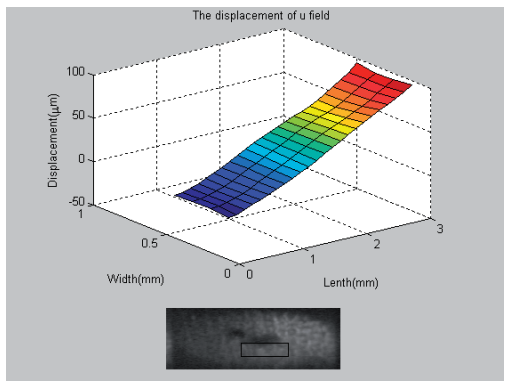

(a)

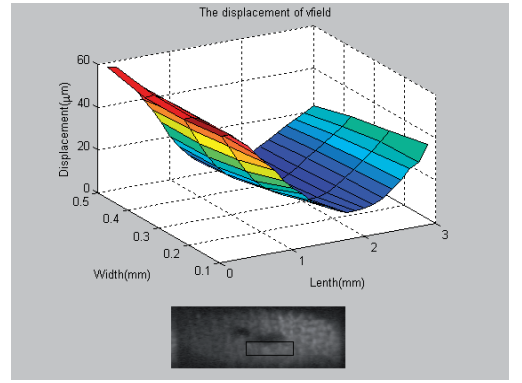

(b)

Figure 5. Displacements of $\mathrm{u}$ and $\mathrm{v}$ fields in $50 \mu \mathrm{s} .60 \mu \mathrm{s}$.

The impact experiments are performed in an SHTB. The single-hole and dual holes samples with the aperture size of $0.8 \mathrm{~mm}$ (as shown in fig.4 and fig.5) are made of aluminum alloy named LY12 and their surfaces are mildly polished to increase the intensity of reflected light so that a good exposure can be obtained.

Besides, to satisfy the exposure requirements the camera is not perpendicular to the sample surface hence the slope angle has to be adjusted in image processing. The image resolution ratio is about $50 \mu \mathrm{m}$ and the accuracy of measurement displacement is $0.5 \mu \mathrm{m}$. The accuracy of measuring displacement is determined by the image-processing code design. The correlation algorithm we use can reach the accuracy of $0.5 \mu \mathrm{m}$. While the image resolution is determined by the structure of the image-forming system (the high-speed camera and the CCD) and our system can only reach $50 \mu \mathrm{m}$.

The image processing includes mainly three steps, as follows,

1. Image cutting. Because each CCD photograph consists of 8 small subgraphs, which depict the object at different times, a method must be employed to divide the photograph accurately. In this paper, a method based on correlation calculation is presented, and it is used in a specially written code with MATLAB v6.0. When we select an interesting region in the first small picture, the other 7 pictures can be obtained automatically and saved as different files. Because the successive pictures are defined by the codes based on Statistic Correlation Method, it can be considered that the rigid displacement has been erased in the area selection.

2. Image pre-processing. In this step, the gray-degree difference and rigid motion among 8 small pictures resulted from measuring system error are taken out.

3. Displacement calculating. The calculation is based on the correlation iteration, as depicted in the above equations. 


\section{RESULTS AND DISCUSSIONS}

The dynamic deformation of single-hole sample under impact tensile test is shown in fig.4 (b), while the fig.4 (a) displays the state when the sample is not loaded and the 8 subgraphs are the same to check the stability of the optical measuring system. The black spot on the left upper of the photograph is the inherent flaw and it is always the same during the dynamic tensile process.

From fig.4 (b), it is obviously shown that the hole located in the middle of the sample is gradually extended according to the tensile process. The displacements fields of a sample at different times can be acquired quantitatively from 8 subgraghs which are extracted from a frame CCD photograph.

For instance, in the time interval of $50 \sim 60 \mu$ s, at a region under and close to the single hole (marked by a black rim in the subgraphs), the displacement field along the tensile direction $u$, is shown in fig.5(a), obtained by using a novel image processing method and the digital correlation algorithm. However, the lateral displacement $\mathrm{v}$ is shown in fig. 5(b). It is found from the experimental results that in the region we concern the deformation field $u$ arises in a monotonic and uniform way while the deformation field $\mathrm{v}$ is symmetrically distributed around the central axis of the hole.

In the same way, the deforming evaluation of dual-adjacent-holes sample is acquired, shown as fig.6 (b) and the fig. 6(a) is shot for verify the reliability of the measuring system.

By image processing based on correlation algorithm described in the above, the displacements in different time interval are obtained. The fig.7 shows the deformation fields of $u$ and $v$ components in the concerned area (the right region of the dual-adjacent-holes marked with the black rim) from $40 \mu \mathrm{s}$ to $50 \mu \mathrm{s}$ after tensile process. The results reveal that these displacements fields are more complicated

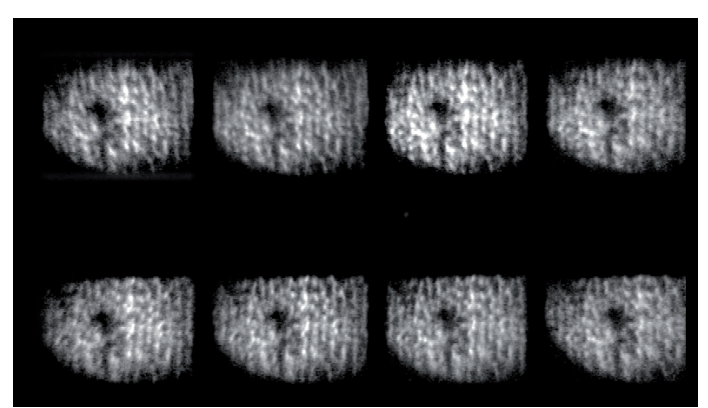

(a)

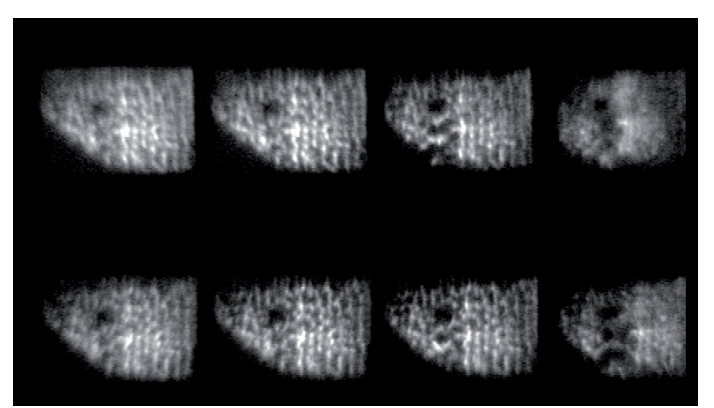

(b)

Figure 6. Images of dual holes sample before and after loading.

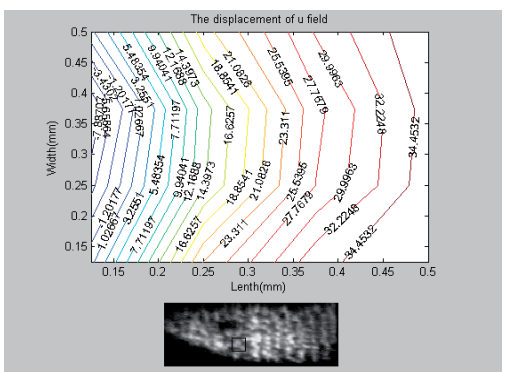

(a)

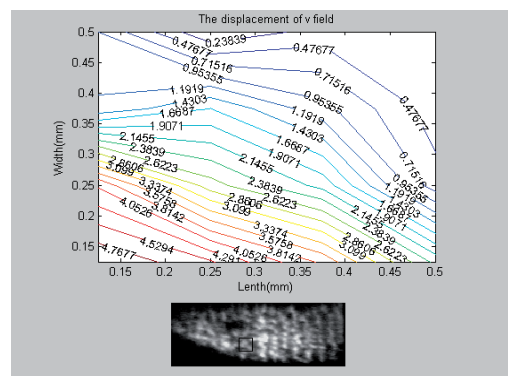

(b)

Figure 7. Contour map of $\mathrm{u}$ and $\mathrm{v}$ fields in $40 \mu \mathrm{s} .50 \mu \mathrm{s}$. 


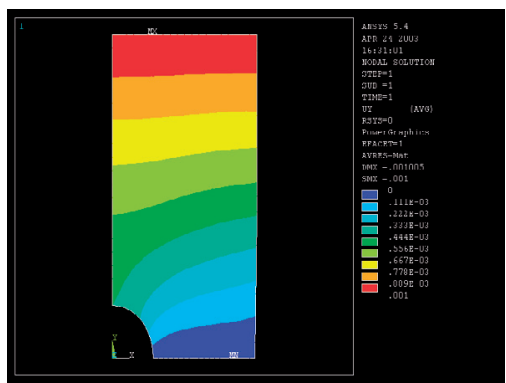

(a)

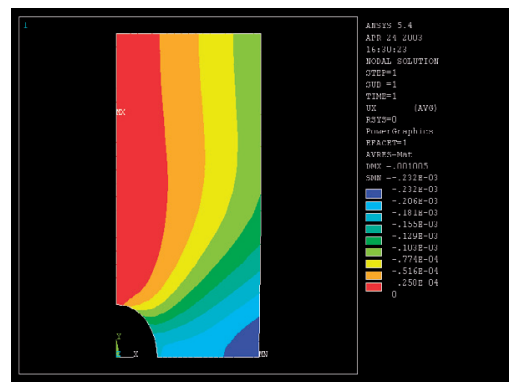

(b)

Figure 8. Numerical simulation of $u$ and $v$ fields of single-hole sample.

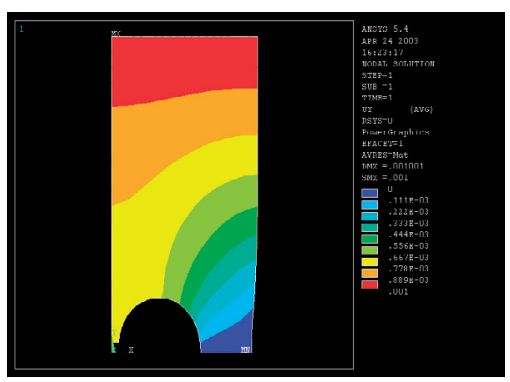

(a)

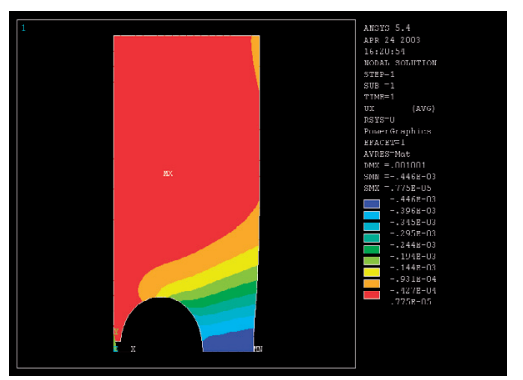

(b)

Figure 9. Numerical simulation of $u$ and $v$ fields of dual-holes sample.

than those of single-hole sample. Particularly, the u field according to the tensile direction still arises in a monotonic way while the field has cove form.

To be illustrated, the displacements achieved in the above experiments are the relative deformation to a single reference point and in this case the successive derived strain fields are more convenient to use. Compared with the numerical simulation results (shown in the below), the qualitative identification can be concluded from the tendency and the degree of closeness of the contour lines.

In this paper the LSAIDYNA module of ANSYS 8.0 is used in numerical simulation and the results of single hole and dual-adjective-holes samples are shown in fig.8 and fig.9. Compared with fig.5 and fig.7 individually, the results obtained by numerical simulation are uniform with the ones acquired by experiments. It shows that the new dynamic measuring system is effective and has a wide application prospect.

\section{CONCLUSION}

In this paper, the high velocity deformations of two different kinds of hole-excavated dog-bone samples are investigated by a newly developed optical measuring system, including analysis software based on correlation calculation and iteration theory, with a resolution of $50 \mu \mathrm{m}$ and an accuracy of $0.5 \mu \mathrm{m}$. The experimental observations are reasonable and fit the numerical simulation quantitatively. It is an attempt and offers a new look-angle to reveal the details in dynamic damage mechanism by experimental facility. 


\section{References}

[1] Papanikos P., Meguid S. A., Three-dimensional Finite Element Analysis of Cold Expansion of Adjacent Holes. International Journal of Mechanical Sciences 40 (1994) 1019-1028

[2] Kim Cheol, Kim Dae-Jin. Finite Element Analysis of the Residual Stress by Cold Expansion Method under The Influence of Adjacent Holes. Journal of Materials Processing Technology 153 (2004) 986-991

[3] Jian Zheng, Zeping Wang, Chengyou Sun. Damage Evolution in Ductile Materials under Intense Dynamic Loading, Acta Mechanica Solida Sinic 16 (1995) 336-343

[4] Lizhu Lliu, Changsuo Zhang, Jizhong, Jia. Void Growth Model of Material Damage and Fracture. Journal of Taiyuan University of Technology 34 (2003) 504-509

[5] Chengguang huang, Siying Chen. Real-Time Measurement on Deformation Fields of Notched Samples Under Impact Tension. International Journal of Impact Engineering 31 (2005) 329 339

[6] Jin G.C., Computer-aided optical metrology (1997) pp 148 